\title{
Unliganded thyroid hormone receptor inhibits formation of a functional preinitiation complex: implications for active repression
}

\author{
Joseph D. Fondell, Ananda L. Roy, and Robert G. Roeder \\ Laboratory of Biochemistry and Molecular Biology, The Rockefeller University, New York, New York 10021 USA
}

\begin{abstract}
The thyroid hormone receptor (TR) belongs to the steroid/nuclear receptor superfamily of ligand-inducible transcription factors. Numerous studies using transient transfection assays have demonstrated that in the absence of thyroid hormone $\left(T_{3}\right)$, unliganded TR acts as a constitutive repressor of transcription on genes bearing TR-response elements. We examined the molecular mechanism of TR repression in vitro using both HeLa nuclear extracts and purified basal factors. Here, we show that unliganded TR is an active transcriptional repressor, distinct from passive repressors that compete with activators for DNA binding. Repression by TR can be relieved by adding the $T_{3}$ analog triiodothyroactic acid, suggesting that liganded TR undergoes a conformational change that masks or disrupts the repressor function. Repression by TR is mediated through the basal transcription machinery and can occur independently of previously characterized TATA-binding protein-associated cofactors thought to be involved in either basal repression or activator-dependent transcription. TR inhibits transcription at an early step during preinitiation complex (PIC) assembly, as preassembled PICs are refractory to the inhibitory effects of TR.
\end{abstract}

[Key Words: Thyroid hormone receptor; active repression; in vitro transcription; preinitiation complex]

Received March 25,1993; revised version accepted May 6, 1993.

Initiation of transcription on protein-encoding genes involves the ordered assembly of RNA polymerase II and a set of general initiation factors (TFIIA, -B, -D, -E, -F, -G/J, $-\mathrm{H}$ and, in some cases, $-\mathrm{I}$ ) into a functional preinitiation complex (PIC) on minimal promoter elements (TATA and initiation region) (for review, see Roeder 1991; Zawel and Reinberg, 1992). Activation and repression of transcription can be achieved by gene-specific factors that bind to control elements upstream of the minimal promoter and regulate the assembly and function of the PICs (for review, see Levine and Manley 1989; Mitchell and Tjian 1989; Ptashne and Gann, 1990; Renkawitz 1990). The effects of these upstream factors may require additional positive or negative cofactors, some of which bind transiently or stably to the TATA-binding protein (TBP) (Hoffmann et al. 1990; Pugh and Tjian 1990; Dynlacht et al. 1991; Meisterernst and Roeder 1991; Meisterernst et al. 1991; Tanese et al. 1991; Inostroza et al. 1992; Zhou et al. 1992).

The thyroid hormone receptor (TR) is a member of the steroid/nuclear receptor superfamily, a class of proteins best characterized as ligand-inducible transcriptional activators (for review, see Evans 1988; Beato 1989). TR can regulate transcription in two ways: In the presence of thyroid hormone (triiodothyronine, or $T_{3}$ ), the receptor activates transcription from genes bearing TR-response elements (TREs). In the absence of $\mathrm{T}_{3}$, the receptor still binds DNA but acts as a transcriptional repressor of genes bearing TREs (Brent et al. 1989a; Damm et al. 1989; Graupner et al. 1989; Sap et al. 1989; Baniahmad et al. 1990, 1992). In addition, TR repression in the presence of ligand has also been reported (Krishna et al. 1989; Hudson et al. 1990). The dual role as activator-repressor by TR presumably broadens the range of regulation mediated by $\mathrm{T}_{3}$, a hormone required for a vast array of biological responses, including growth, development, and differentiation (Samuels et al. 1988).

In general, two different mechanisms have been proposed to explain transcriptional repression by TR. The first mechanism involves a passive repression in which TR simply blocks the binding of activators or basal factors to the promoter by competition for binding sites (Glass et al. 1988; Graupner et al. 1989; Krishna et al. 1989; Hudson et al. 1990). Similarly, because TR can form heterodimers with other nuclear receptors, another form of passive repression may involve the formation of inactive heterodimers (Forman et al. 1989; Glass et al. 1989). The second mechanism proposed for TR repression involves active repression or silencing (Renkawitz 1990; Jaynes and O'Farrell 1991). In this model, unliganded TR counteracts the effects of activators bound at independent sites by direct (inhibitory) interactions with 
the general initiation factors, the activators themselves, or cofactors required for communication between them. Although mechanisms for active repression are poorly understood, Baniahmad and colleagues (1992) have recently identified a putative silencing domain on the TR thought to be important for active repression as assayed by transient transfection analyses.

Here, we report studies of the molecular mechanism of TR repression using cell-free transcription assays. We show that the unliganded form of TR is an active repressor of transcription in vitro, that the target of TR repression is the basal transcription machinery, and that repression involves inhibition of an early step in PIC assembly. It is demonstrated further that repression by TR is alleviated by the $T_{3}$ analog triiodothyroacetic acid (TRIAC), consistent with the hypothesis that liganded TR undergoes a conformational change that masks or disrupts the repressor function and possibly potentiates an activator function.

\section{Results}

$T R$ repression of rat growth hormone promoter in vitro

As a source of TR for our studies, we expressed the human TR $\alpha 1$ cDNA (Nakai et al. 1988) in Escherichia coli as a fusion protein with 10 histidine residues at the amino terminus. TR was subsequently purified using $\mathrm{Ni}^{2+}$-chelate NTA column chromatography under nondenaturing conditions (Hoffmann and Roeder 1991). The expressed TR protein bound efficiently and specifically to a synthetic palindromic TRE as determined by both footprint and electromobility-shift assays /data not shown). We first asked whether TR would repress transcription in vitro from a wild-type promoter containing a natural TRE. The rat growth hormone $(\mathrm{rGH})$ promoter is regulated by $T_{3}$ and contains a natural TRE between positions -188 and -165 (Fig. 1A) that consists of three TR-binding half-sites with consensus sequence AGGT(C/A)A (Brent et al. 1989b). It has been reported that unliganded TR will repress basal transcription from the $\mathrm{rGH}$ promoter in a transient transfection assay (Brent et al. 1989a). Figure 2 shows that purified recombinant TR repressed transcription in vitro $\geqslant 10$-fold from $\mathrm{rGH}$ 236 (lane 2), a rGH promoter template containing wildtype sequences between -236 and the transcription start site (Fig. 1A). That the repression was specific for the TRE-containing $\mathrm{rGH}$ promoter is shown in three ways. First, a reference template containing no TREs $(2 \times$ Spl HIV CAT; Fig. 1A) was unaffected by TR (Fig. 2). Second, the repression on rGH-236 was competed by the addition of a molar excess of a TRE oligonucleotide (lane 3). Third, the $\mathrm{rGH}$ promoter deletion template $\mathrm{rGH}-137$ (Fig. 1A), which lacks the natural TRE, was repressed far less effectively than rGH-236 (Fig. 2, lanes 4,5). The modest TR repression observed on the $\mathrm{rGH}-137$ template may have been the result of a second weaker TRE near the rGH TATA element (Kim et al. 1992). Thus, unliganded TR can repress transcription in vitro from a promoter normally regulated by TR in vivo.
$T R$ repression of synthetic TRE-linked promoters in vitro

We then asked whether TR could repress transcription from a series of heterologous promoters containing synthetic TRE inserts. Toward this end, we inserted synthetic palindromic TREs /consensus TCAGGTCATGACCTGA; Glass et al. 1988) into various sites within the human immunodeficiency virus-1 (HIV-1) promoter, the herpes thymidine kinase $(\mathrm{TK})$ promoter, and the adenovirus major late (AdML) promoter (Fig. 1A,B). We first examined whether TR could repress activated transcription from TRE-linked promoters containing functional activator-binding sites. Figure 3 (lanes 7,8 ) shows that TR repressed transcription in vitro sevenfold from a TRE-linked TK promoter /containing wild-type TK positions -105 to +51 ; Fig. 1A), in accordance with previous observations from cellular transfection studies (Damm et al. 1989; Baniahmad et al. 1990). Similarly, TR repressed transcription 5- or 14-fold from an HIV-80 promoter (containing two Sp1 sites; Fig. 1A) with one or two TRE inserts, respectively (Fig. 3, lanes 1-4). These findings indicate that TR can block or counteract Spl-activated transcription. Transcription from the reference promoter lacking TREs was not repressed by TR, indicating that the repression was specific.

Interestingly, when a single TRE was placed on an HIV-38 minimal promoter (lacking Spl sites; Fig. 1A), TR repressed transcription 45-fold (Fig. 3, lanes 5,6), strongly suggesting that the target of the TR repression is the basal transcription machinery. The high basal level of transcription observed on the HIV-38 minimal promoter (in comparison with the HIV-80 Spl-dependent transcription; cf. lanes 1 and 5) reflects a fourfold increase in template concentration (see Fig. 3 legend), as at comparable low template levels the HIV-80 templates showed a sixfold higher activity than the HIV-38 template (data not shown).

Experiments with TRE-linked AdML promoters (Fig. 1B) gave similar results. TR repressed transcription eightfold from an AdML promoter containing wild-type sequences from -404 to +10 and a single TRE inserted into the upstream stimulatory factor (USF) site at position -60 (see Fig. 1B and 4A, lanes 1,2). Again, the repression was specific for the TRE-linked promoter as neither an AdML promoter containing a mutated TRE (Fig. 4A, lanes 3,4) nor a wild-type AdML reference promoter was significantly affected by TR. Surprisingly, TR repressed transcription (although slightly less effectively) when the TRE was as far as $415 \mathrm{bp}$ from the AdML transcription start site (Fig. 4, lanes 5,6), indicating that TR repression is still efficient over long distances. When one or more TREs were linked to an AdML minimal promoter lacking upstream activator-binding sequences ( $\triangle 53 \mathrm{ML}$; Fig. 1B), TR abolished transcription completely (Fig. 4A, lanes 7-10). This finding strengthens the notion that TR targets the basal transcription machinery for repression.

It could be argued that the synthetic palindromic TRE on these constructs provides a binding site for a tran- 


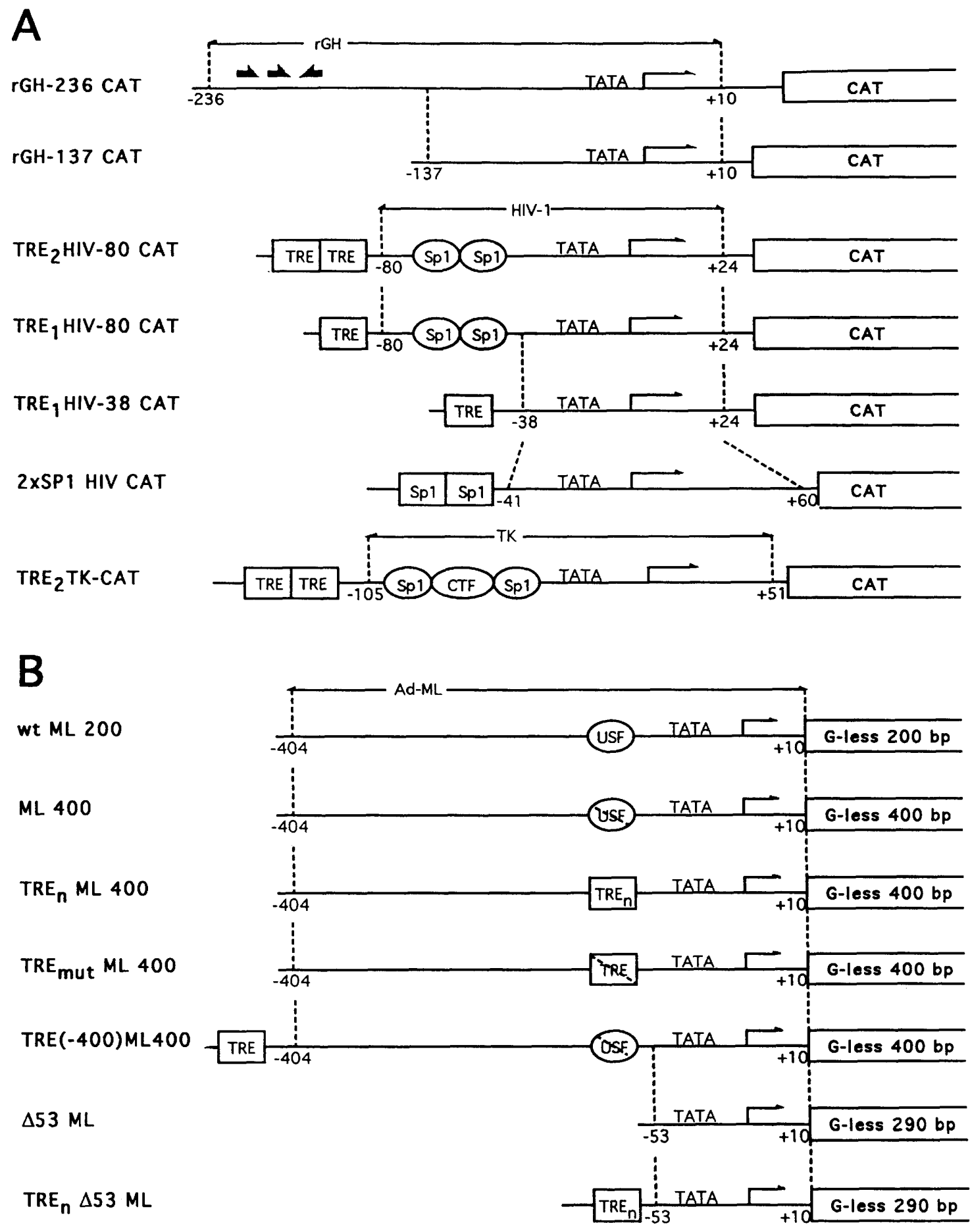

Figure 1. Schematic representation of promoters used for in vitro transcription assays. $(A)$ Templates analyzed by primer extension assay. Templates containing various regions of the rGH, HIV-1, and TK promoters are indicated. Squares indicate synthetic binding sites for transcription factors; ovals indicate natural binding sites. TRE denotes the synthetic palindromic-binding site for TR (TCAGGTCATGACCTGA; Glass et al. 1988). Solid half arrows above the rGH promoter denote a natural TRE (Brent et al. 1989b). (B) Templates analyzed by G-less cassette assay. Templates containing various regions of the AdML promoter are indicated. Squares and ovals are as above; symbols containing a dashed line indicate that the binding site has been mutated (see Materials and methods). $\mathrm{TRE}_{\mathbf{n}}$ indicates a promoter containing 1,2, or 3 TRE inserts.

scriptional activator present in the HeLa nuclear extract. Accordingly, TR may have inhibited in vitro transcrip- tion passively by simply competing with the activator for binding at the TRE. This model predicts that liganded 


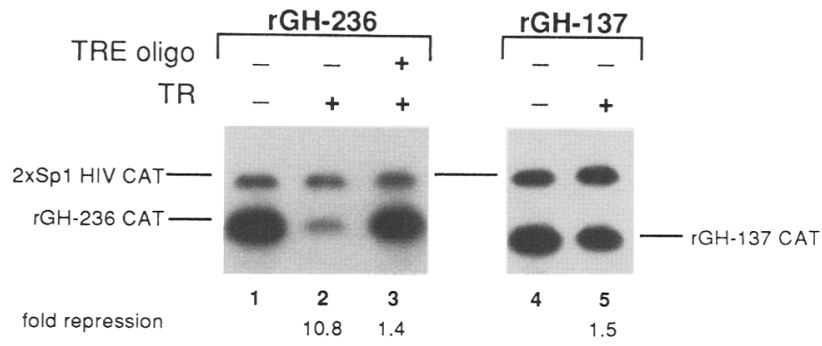

Figure 2. TR repression of the rGH promoter in vitro. Reactions $(25 \mu \mathrm{l}$ ) contained $50 \mathrm{ng}$ of the rGH-236 template (lanes $1-3$ ) or $100 \mathrm{ng}$ of the rGH-137 template (lanes 4,5 ) with $50 \mu \mathrm{g}$ of HeLa nuclear extract under standard conditions. TR (250 ng) or a synthetic palindromic TRE oligonucleotide (50 ng) was added as indicated. Each reaction contained $10 \mathrm{ng}$ of the $2 \times$ Sp1HIVCAT template as an internal control. Transcripts were analyzed by primer extension; extension products from test and reference templates are indicated.

TR would repress transcription in vitro as well as for better than) unliganded TR, as ligand has been shown to enhance TR binding to a synthetic palindromic TRE (Ribeiro et al. 1992). To test this hypothesis, we added the $T_{3}$ analog TRIAC to the transcription reaction (Fig. $4 \mathrm{~A}$, lane 11). In the presence of ligand, TR did not repress transcription, thus suggesting a mechanism involving active repression. In addition, this result suggests that the liganded form of TR undergoes a conformational change that alleviates the TR active repression. To verify further that TR represses in an active rather than in a passive manner, we compared the basal transcription levels with equimolar quantities of AdML promoters containing or lacking TREs (Fig. 4B). If the TRE acts as a binding site for an endogenous HeLa cell activator, a higher basal signal would be expected from AdML promoters containing TRE inserts in the absence of TR. Clearly this was not the case (Fig. 4B), as AdML promoter constructs containing as many as three TREs gave a basal signal that was essentially equivalent to promoters containing none.

Taken together (Figs. 3 and 4), these results show that unliganded TR can function as an active repressor of transcription in vitro. Furthermore, it appears improbable that the target of repression is an activator binding at either the TRE or an independent site. Rather, we infer from these data that TR may target the basal transcrip- tion machinery, as repression is much more effective on minimal promoter elements.

\section{$T R$ repression in a reconstituted transcription assay: active repression through basal factors}

If TR targets the basal transcription machinery for active repression, it is predicted that TR will repress transcription in a reconstituted assay consisting minimally of highly purified basal transcription factors. Therefore, we tested the activity of TR in a purified system consisting of recombinant TATA-binding protein (TBP or TFIID $\tau$ ), recombinant TFIIB, and purified fractions containing TFIIA, TFIIE/F/H, and RNA polymerase II. As expected, TR repressed transcription effectively from a TRE-linked minimal AdML promoter (Fig. 5, lane 2), strongly supporting our supposition that TR interacts negatively with the basal transcription machinery. Because the overall repression by TR on the $\mathrm{TRE}_{3} \Delta 53 \mathrm{ML}$ template was greater in a nuclear extract (Fig. 4A, lane 10, complete repression) versus the purified system (Fig. 5, lane $2 ; 7.3$-fold repression), the possibility exists that an additional nuclear factor (absent from the purified system) may serve to stabilize or enhance TR repression. However, the data in Figure 5 is significant because it suggests that TR has an intrinsic ability to repress transcription independently of the previously characterized TBPinteracting negative cofactors, $\mathrm{NC1}$ and $\mathrm{NC2}$ (DR1), which were shown to repress basal transcription on AdML promoters using a reconstituted system highly analogous to the one used here (Meisterernst and Roeder 1991; Meisterernst et al. 1991; Inostroza et al. 1992). Furthermore, TBP-associated factors in natural TFIID (Hoffmann et al. 1990; Pugh and Tjian 1990; Dynlacht et al. 1991; Tanese et al. 1991; Zhou et al. 1992) and other soluble cofactors (Meisterernst et al. 1991) required for activator-dependent transcription appear to be dispensable for TR-mediated repression, as defined by this in vitro assay. Thus, these results (1) further define the minimal components that are necessary and sufficient for TR repression in vitro, and (2) suggest that TR might interact directly (negatively) with the basal transcription initiation factors.

The data presented above suggest that TR repressed transcription in the reconstituted system as a monomer or homodimer. However, recent studies have shown that the retinoid X receptors $(\mathrm{RXR})$, also members of the ste-

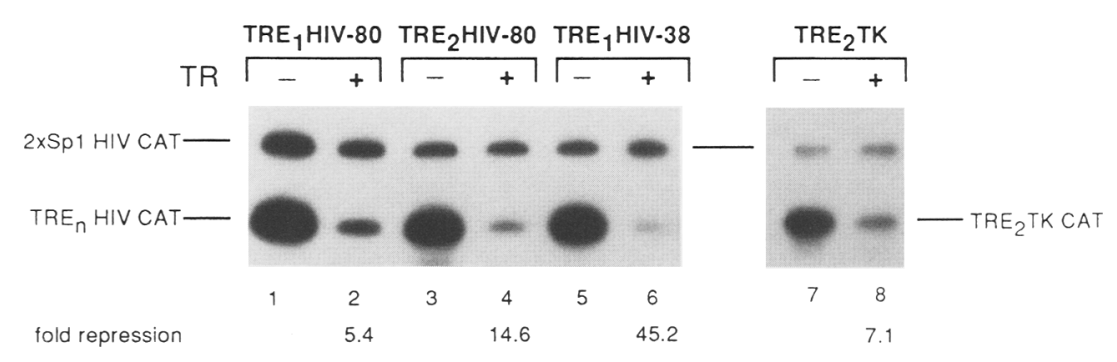

Figure 3. TR repression of the HIV-1 and TK promoters in vitro. Reactions $(25 \mu l)$ contained either $50 \mathrm{ng}$ of the TRE $\mathrm{T}_{1}$ HIV-80CAT template (lanes 1,2 ), $50 \mathrm{ng}$ of the $\mathrm{TRE}_{2} \mathrm{HIV}$ 80CAT template (lanes 3,4 ), $200 \mathrm{ng}$ of the $\mathrm{TRE}_{1}$ HIV-38CAT template (lanes 5, 6), or 400 ng of the TRE ${ }_{2}$ TKCAT template (lanes 7,8 ). Each reaction contained $50 \mu \mathrm{g}$ of HeLa nuclear extract and $10 \mathrm{ng}$ of the internal control template $2 \times$ SplHIVCAT. TR ( $250 \mathrm{ng}$ ) was added as indicated. Transcripts were analyzed by primer extension. 
Figure 4. Transcriptional regulation of the AdML promoter by TR in vitro. $(A)$ TR repression of the AdML promoter in vitro is DNA-binding specific and can be relieved by the $\mathrm{T}_{3}$ analog TRIAC. Reactions $(25 \mu \mathrm{l})$ contained $200 \mathrm{ng}$ of the appropriate AdML promoter template (indicated above the lanes| with $50 \mu \mathrm{g}$ of HeLa nuclear extract under standard conditions. Each reaction contained $33 \mathrm{ng}$ of the wt ML200 template as an internal control. TR $(250$ $\mathrm{ng}$ ) or TRIAC ( $20 \mu \mathrm{M}$ final concentration) was added as indicated. Transcripts were analyzed using the G-less cassette assay. $(B)$ HeLa nuclear extract lacks an activator that acts in vitro through a palindromic TRE. Reactions $(25 \mu$ l) contained $200 \mathrm{ng}$ of the appropriate AdML promoter template lacking or containing TREs (indicated above each lane) with $50 \mu \mathrm{g}$ of HeLa nuclear extract under standard conditions. Each reaction contained $50 \mathrm{ng}$ of the wt ML200 template as an internal control. No TR was added to these reactions. Transcripts were analyzed using the G-less cassette assay.

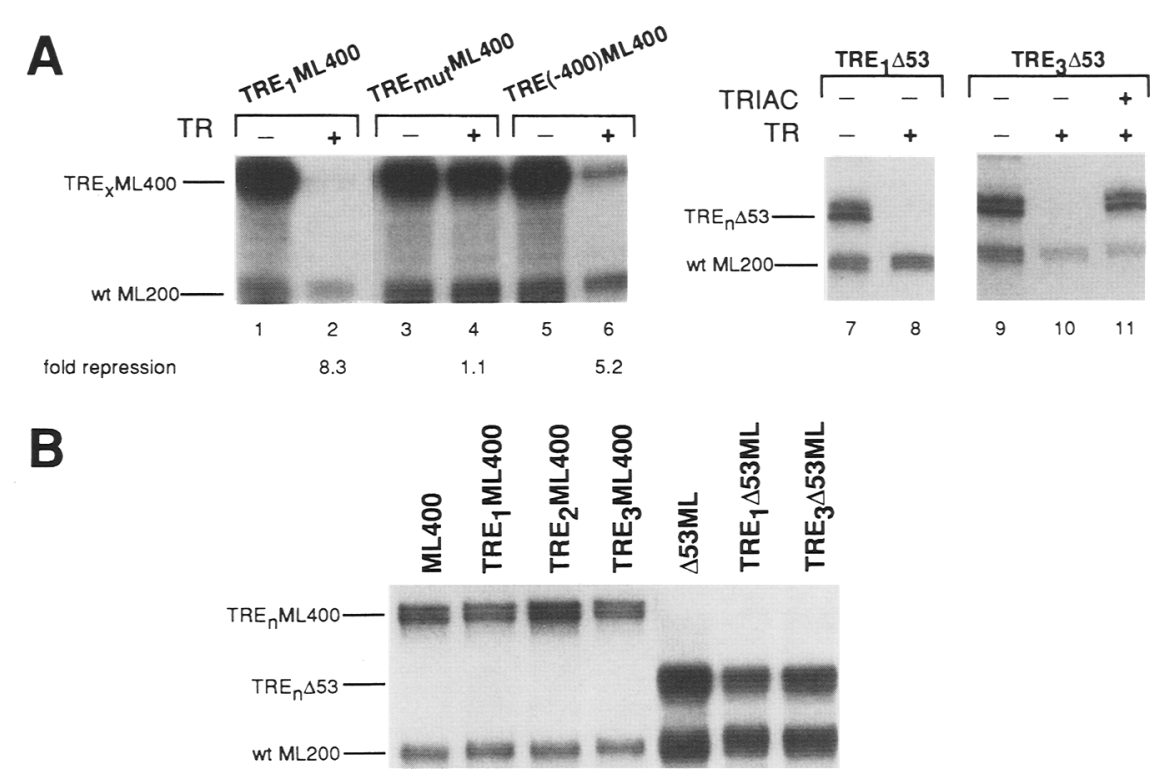

roid/nuclear receptor superfamily, markedly enhance the binding of TR to a TRE in vitro by forming a TRRXR heterodimer (Yu et al. 1991; Kliewer et al. 1992; Leid et al. 1992; Marks et al. 1992; Zhang et al. 1992). Moreover cotransfection experiments indicate that RXR can enhance transcriptional activation by TR (in the presence of $\mathrm{T}_{3}$ ) in vivo (Yu et al. 1991; Marks et al. 1992; Zhang et al. 1992). We asked, therefore, whether RXR might enhance or relieve TR repression in the reconstituted transcription assay. With this objective, we purified mouse RXR $\beta$ (mRXR $\beta$ ) from baculovirus-infected Sf9 cells expressing the mRXR $\beta$ cDNA. Consistent with the previous reports, baculovirus-expressed $\mathrm{mRXR} \beta$ protein enhanced the binding of TR to a palindromic TRE, as assayed by an electromobility-shift assay (data not shown|. However, addition of RXR $\beta$ to the system reconstituted with general factors had no effect on TR repression (Fig. 5, lane 3). Conversely, addition of TRIAC again relieved the repression mediated by TR (lane 4) or by TR plus RXR (data not shown). These results suggest that in our reconstituted transcription system, TR can repress transcription actively either as a TR homodimer or as a TR-RXR heterodimer. Moreover, because RXR $\beta$ is naturally present in HeLa cells (Leid et al. 1992), it appears likely that the active repression demonstrated above using HeLa nuclear extracts (Fig. 2-4) is mediated by unliganded TR-RXR heterodimers. Although it remains possible that RXR may contribute to the repression activity of TR, the results of Figure 5 indicate that TR can repress transcription independently of other nuclear receptors. The transcriptional activation by TRRXR heterodimers observed in vivo presumably requires ligand for TR or RXR (or both) in addition to other cofactors (see Discussion).

\section{$T R$ inhibits functional PIC assembly}

Active repression by TR through the basal transcription machinery might involve inhibition of (1) PIC formation, (2) transcriptional initiation (PIC function), or (3) transcriptional elongation. In an effort to identify the step at which TR represses transcription, we performed singleround initiation experiments. In these assays, PICs were allowed to assemble on the $\mathrm{TRE}_{1}$ ML400 promoter by preincubating the template in nuclear extract for $25 \mathrm{~min}$ (Hawley and Roeder 1985). After the preincubation, $0.025 \%$ Sarkosyl (a nonionic detergent) and NTPs were added to initiate transcription. This low level of Sarkosyl (a non-ionic detergent) does not disrupt any preformed

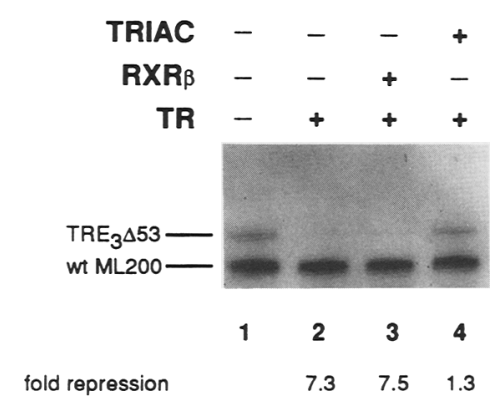

Figure 5. Repression by TR in vitro in a reconstituted system. Each reaction contained $100 \mathrm{ng}$ of the $\mathrm{TRE}_{3} \Delta 53 \mathrm{ML}$ template and $33 \mathrm{ng}$ of the wt ML200 template as an internal control. Reactions were performed using recombinant and highly purified general factors (see Materials and methods). TR (250 ng), RXR $\beta$ ( $50 \mathrm{ng}$ ), or TRIAC ( $20 \mu \mathrm{M}$ final concentration) was added as indicated. Transcripts were analyzed using the G-less cassette assay. 
PICs, but does prevent reassembly of PICs and reinitiation by RNA polymerase II (Hawley and Roeder 1987). If TR acts by blocking initiation or elongation, addition of TR after formation of a PIC should repress transcription. As shown in Figure 6B, TR repressed transcription when added either $10 \mathrm{~min}$ before (lane 2) or at the beginning (lane 3) of PIC assembly. However, when TR was added after PIC formation (lane 4), no repression was observed. These data strongly suggest that TR inhibits the formation of a functional PIC but not the subsequent initiation or elongation stages of transcription. Furthermore, addition of TRIAC after TR was preincubated with template and nuclear extract did not relieve the repression (lane 6). This suggests either that TR is no longer subject to a ligand-induced conformational change after interactions with the basal transcription machinery or that TR introduces an irreversible (e.g., conformational) change in one of the components thereof. To eliminate the possibility that TR represses initiated complexes that might have resulted from trace quantities of NTPs in the nuclear extract, we repeated the experiment with purified basal factors. Figure $6 \mathrm{C}$ again shows that TR repressed transcription only when added before PIC formation but not after.

Active repression by TR during PIC formation was verified further by a third set of experiments. PICs were formed on a TRE 2 HIV-80CAT promoter by preincubating the template in nuclear extract. The assembled PICtemplates were then purified by gel filtration (Bio-Gel A-1.5m/, and transcription was initiated by adding a limiting set of nucleotides (GTP, UTP, CTP) lacking ATP. Because there are no A nucleotides encoded in the first 13 bases of the HIV-1 mRNA transcript, this assay restricts synthesis to a short 13-nucleotide transcript (13- mer) (Kato et al. 1992). In agreement with the results in Figure 6, TR repressed the synthesis of the 13-mer when added before (or at the beginning of) PIC assembly (Fig. $7 B$, lane 3). Importantly, this result again confirms that TR does not effect pausing or block elongation of initiated complexes in a promoter-proximal fashion. As predicted, TR did not repress 13-mer formation when added after PIC-template formation and purification (lane 4). Addition of a TRE oligonucleotide concomitantly with TR before PIC formation relieved the repression, indicating that the repression was specific (lane 5). The additional band at $15 \mathrm{bp}$ and the fainter bands at 17 and $19 \mathrm{bp}$ (observed in Fig. 7B) are probably the result of contaminating ATP in the gel-purified PIC-template eluate.

We conclude from these studies that TR negatively affects the formation of a functional PIC. That TR does not inhibit the function of a fully assembled PIC is evidenced by the failure of TR to repress transcription in vitro when added to a reaction after PIC formation. Moreover, these results indicate that TR does not negatively affect the subsequent initiation or early elongation steps of transcription from a preformed PIC.

\section{Direct interaction of $T R$ with the basal transcription machinery}

On the basis of our findings that TR targets the basal transcription machinery for repression, it is conceivable that TR interacts directly with one or more of the basal transcription factors. To investigate this possibility, immunoprecipitation experiments were carried out with in vivo ${ }^{35} \mathrm{~S}$-labeled TR and purified recombinant basal factors. Figure 8 shows that a TR-TFIIB complex could be detected in solution using polyclonal antisera against

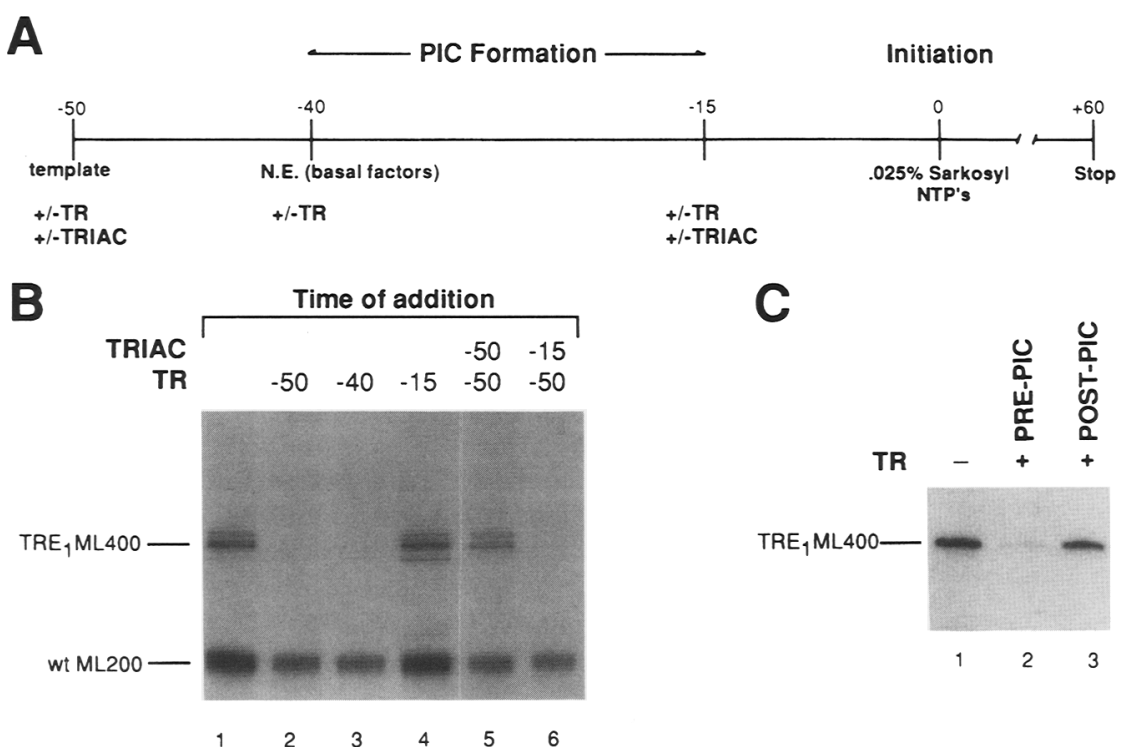

ng) was added either before (PRE-PIC) or after (POST-PIC) the preincubation, as indicated a quently initiated upon the addition of NTPs, and transcripts were analyzed using the G-less cassette assay. Sarkosyl was not added to these reactions.
Figure 6. TR inhibits formation of a functional PIC. (A) Experimental scheme for single-round initiation assay. (B) Singleround transcriptional initiation assay. In each reaction, the $\mathrm{TRE}_{1} \mathrm{ML} 400$ template (100 ng) was preincubated with $50 \mu \mathrm{g}$ of HeLa nuclear extract for $25 \mathrm{~min}$ to allow PIC formation. Sarkosyl $10.025 \%$ final concentration) and NTPs were then added, and the reactions were allowed to proceed for $50 \mathrm{~min}$. TR ( $250 \mathrm{ng}$ ) or TRIAC (final concentration $20 \mu \mathrm{M}$ ) was added at the indicated times shown above the lanes. Each reaction contained $33 \mathrm{ng}$ of the wt ML200 plasmid as an internal control. Transcripts were analyzed usi ng the G-less cassette assay. $(C)$ Reconstituted transcription assay. In each reaction, the $\mathrm{TRE}_{1} \mathrm{ML} 400(100 \mathrm{ng})$ template was preincubated at $30^{\circ} \mathrm{C}$ with both recombinant and purified general factors (as described in the legend to Fig. 5) in the absence of NTPs for $25 \mathrm{~min}$. TR $(250$ 


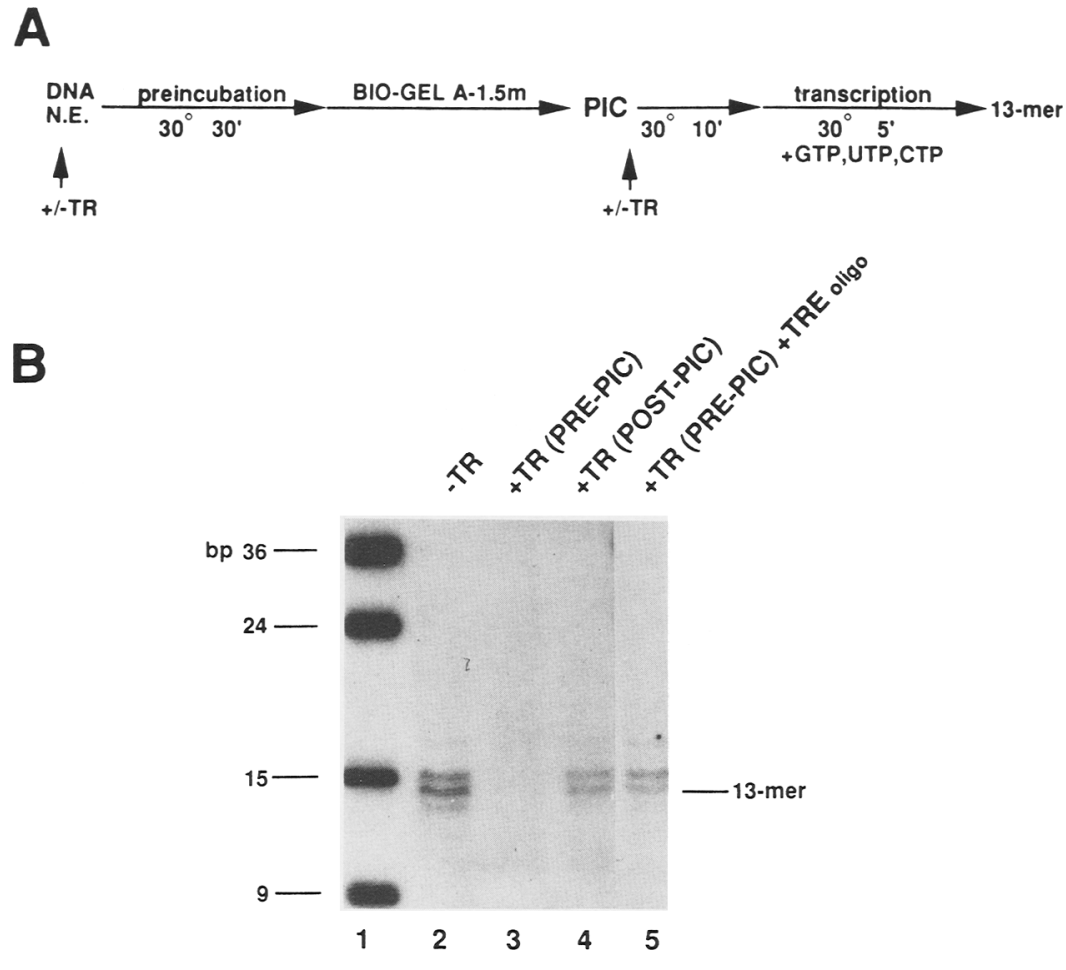

Figure 7. TR inhibits formation of a functional PIC as assayed by PIC-template purification. (A) Experimental scheme for PIC-template purification and subsequent transcriptional initiation. $(B)$ In each reaction, the test template TRE $_{2}$ HIV-80CAT (100 ng) was preincubated in $50 \mu \mathrm{g}$ of HeLa nuclear extract to allow formation of a PIC. The PIC-template complexes were then purified by gel filtration (Bio-Gel A-1.5m), and transcription was initiated by adding a limited set of NTPs (GTP, CTP, UTP). The absence of ATP results in a prematurely truncated transcript of 13 nucleotides. TR ( $250 \mathrm{ng})$ and a TRE oligonucleotide (100 ng) were added at different times as indicated above each lane.

TFIIB (lane 4). The specificity of this interaction was substantiated by the failure of TFIIB preimmune sera to detect the complex (lane 5). Moreover, TR was unable to form a stable complex with TBP (lane 8). Interestingly, our data are consistent with that of Ing et al. (1992), who demonstrated recently that TFIIB interacts with several

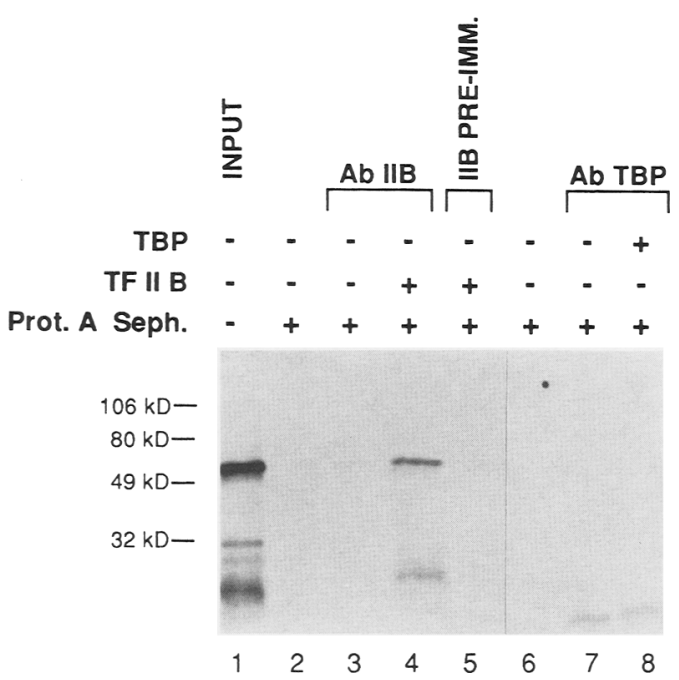

Figure 8. TR interacts directly with TFIIB in solution. In vivo ${ }^{35}$ S-labeled TR ( $50 \mathrm{ng}$ ) was incubated with either TFIIB (200 ng) or TBP $(200 \mathrm{ng})$ as indicated above the lanes. Protein complexes were immunoprecipitated with polyclonal antisera against either TFIIB or TBP as indicated. Immunocomplexes were precipitated with protein A-Sepharose. other members of the steroid/nuclear receptor superfamily. These data raise the question whether TR regulates the basal transcription machinery directly or indirectly by interactions with TFIIB. It should be noted, however, that we have not yet correlated the repressor function of TR with its ability to interact with TFIIB.

\section{Discussion}

The steroid/nuclear receptor TR can function either as a transcriptional activator or as a repressor, depending on the presence of ligand (Damm et al. 1989; Baniahmad et al. 1992 and references therein). On the basis of transient transfection assays, it has been proposed that TR represses transcription by a passive mechanism that involves competition at the level of DNA binding (Glass et al. 1988; Krishna et al. 1989; Hudson et al. 1990). In this report we examined TR repression using cell-free transcription assays. Three lines of evidence are presented that show that TR is an active repressor. First, TR repressed basal transcription through TRE elements inserted into several heterologous promoters. The precise location of the TRE did not appear to be critical, as TR repressed transcription by TREs juxtaposed both to minimal promoters and to promoters with functional activator-binding sites and from distances $\leqslant 415 \mathrm{bp}$. Second, liganded TR did not repress transcription, demonstrating that occupancy of a TRE is not sufficient for repression. Third, promoter constructs containing or lacking TRE inserts gave nearly identical basal transcription signals, indicating the absence of competing TRE-binding activators in a HeLa nuclear extract. Consistent with our 
findings of active repression by TR in vitro, Baniahmad and co-workers (1992) have identified a TR carboxy-terminal transcriptional silencing domain that acts in vivo and can be transferred functionally to a heterologous DNA-binding domain.

In the course of developing an in vitro system to study TR repression, we observed that high doses of test templates were required to obtain quantitatively detectable basal signals, especially on the minimal promoter constructs. Our inability to demonstrate TR activation in this study may be accounted for in part by the high gene doses used, as we could observe TR activation in the presence of TRIAC (albeit very modest) at lower template concentrations (data not shown). Furthermore, optimal activation by TR, and other members of the steroid/nuclear receptor superfamily, may require other soluble factors that might not be present in HeLa cells. For example, transcriptional activation by the retinoic acid receptor (RAR) requires an ElA-like activity present in embryonal carcinoma (EC) cells but not in COS cells (Berkenstam et al. 1992). In addition, optimal activation by TR may be mediated through synergistic protein-protein interactions with other activators and, thus, require precise promoter context of a TRE relative to other activator-binding sites (Voz et al. 1992).

It is conceivable that TR targets either the basal transcription machinery or another activator (binding to an independent site) for active repression. Two major observations presented here show that TR targets the basal transcription machinery. First, TR repressed transcription more efficiently (and, in some cases, completely) from TRE-linked minimal promoter constructs than from TRE-linked promoters containing functional activator-binding sites. Thus, the decreased level of TR repression observed on TRE-linked activator site-containing promoters may be the result of activators partly counteracting TR repression of the basal transcription machinery. These results are also consistent with in vivo data showing repression of TRE-linked minimal promoter reporter genes by TR using transient transfection assays (Baniahmad et al. 1990, 1992). The second observation demonstrating targeting of the basal transcription machinery by TR was provided by the reconstituted transcription assay. In these analyses, TR repressed transcription efficiently from a TRE-linked minimal promoter in a fractionated system consisting of only highly purified basal factors that included recombinant TBP in place of TFIID. This result also rules out competition for binding between TR and an activator, as the system was devoid of both the activators and the various (soluble and TBP-associated) cofactors that are required for activated transcription. Interestingly, we show that $T R$, by virtue of its ability to form a specific complex with TFIIB, can contact directly a component of the basal transcription machinery. It is tempting to speculate that unliganded $\mathrm{TR}$, in a DNA-binding dependent manner, may interact with and freeze TFIIB in a nonfunctional conformation. However, this hypothesis is still under investigation.

In addition, the TR repression mechanism may involve protein-protein interactions that result in the oc- clusion or displacement from the promoter of either a basal transcription factor or an essential cofactor. Alternatively, TR may allow the assembly of all (or some) of the basal factors on the promoter but simultaneously interfere with their normal function. By using an in vitro assay in which progressive steps of transcription were staged, we demonstrated that TR acts to inhibit transcription at an early step during PIC assembly. Interestingly, Johnson and Krasnow (1992) have reported a similar observation in vitro with the Drosophila homeo domain repressor Even-skipped (EVE); relative to the findings shown here, these investigators speculate that EVE may function by directing the assembly of a nonproductive PIC at a minimal promoter. Similarly, transfection assays have revealed that the Drosophila Engrailed (En) protein is an active repressor whose repression domain can be transferred functionally to a heterologous DNA-binding domain (Jaynes and O'Farrell 1991). Finally, the Drosophila protein Krüppel also behaves as an active repressor that can act through minimal promoter elements (Sauer and Jäckle 1991). Therefore, given this precedent for active repression in Drosophila, it is possible that TR represses transcription in a manner mechanistically analogous to the Drosophila homeo domain repressors.

An interesting aspect of the present work is that the repression by TR can be relieved by exposure prior to the $\mathrm{T}_{3}$ analog TRIAC. This observation suggests that liganded TR undergoes a conformational change that alters repressor function. A simpler interpretation holds that the ligand-binding and repression domains are equivalent and that binding of hormone simply masks the repressor function. The work of Baniahmad et al. (1992) is consistent with this theory, as both the silencing domain and the ligand domain are located on the carboxy-terminal end of TR. Moreover, the block of repressor function by ligand may be accompanied by a conformational change that reveals (or potentiates) an activator function. On the other hand, the inability of ligand to relieve repression when added subsequent to incubation of TR with template and the general initiation factors indicates either that TR in the inactive promoter complex is no longer subject to this conformational change or that TR has effected an irreversible change (e.g., a frozen nonproductive conformation or interaction; see above) in one or more components of the general transcription machinery. Further studies will be aimed at elucidating the differential effects of liganded and unliganded TR on specific components of the basal transcription machinery.

\section{Materials and methods}

\section{Preparation of templates}

Construction of rGH-236 (previously called rGH237) and rGH137 was described previously (Larsen et al. 1986; Brent et al. 1989c). Construction of the TRE-linked HIV-1 promoter templates was as follows: $\mathrm{XbaI}$ restriction sites were introduced into the HIV-1 promoter at positions -80 and -38 by PCR 
using the plasmid p-167 (Rosen et al. 1985) as the template. XbaI-BglII restriction fragments containing HIV-1 positions -80 to +24 and -38 to +24 were ligated into $\mathrm{XbaI} / \mathrm{Bg}$ IIIdigested pBLCAT8 + (Luckow and Schütz 1987), giving the plasmids HIV-80CAT and HIV-38CAT. Synthetic palindromic TRE elements (TCAGGTCATGACCTGA; Glass et al. 1988) were cloned into the HindIII sites of these plasmids, giving rise to TRE ${ }_{1}$ HIV-80CAT, TRE ${ }_{2}$ HIV-80CAT, and TRE ${ }_{1}$ HIV-38CAT. The plasmid $2 \times$ Sp1HIVCAT contains two synthetic Sp1 sites and was described previously (Kato et al. 1992). TRE 2 TK-CAT was created by inserting two TRE elements into the BamHI site of pBLCAT8+. The construction of wtML200 (previously called pMLw2) has been described (Pognonec and Roeder 1991). The ML400 construct contains a BglII linker inserted into (and disrupting) the USF-binding site (Pognonec and Roeder 1991). The BglII site of ML400 was used as a convenient and novel cloning site for the palindromic TREs giving rise to $\mathrm{TRE}_{1} \mathrm{ML} 400$, TRE 2 ML400, and $\mathrm{TRE}_{3}$ ML400. Similarly, the cloning of a mutated palindromic TRE element (TCATTTCATGAAATGA) into the BgIII site of ML400 gave rise to $\mathrm{TRE}_{\mathrm{m}}$ utML400. Insertion of a palindromic TRE into the EcoRI site of ML400 gave rise to TRE(-400|ML400. The cloning of one or three TREs into the EcoRI site of $\triangle 53 \mathrm{ML}$ (Sawadogo and Roeder 1985) gave rise to $\mathrm{TRE}_{1} \Delta 53 \mathrm{ML}$ and $\mathrm{TRE}_{3} \Delta 53 \mathrm{ML}$.

\section{Expression and purification of recombinant proteins}

Human TR $\alpha 1$ (Nakai et al. 1988) was introduced into the $10 \mathrm{His}-\mathrm{pET} 1 \mathrm{l}$ vector, and expressed in BL21(DE3) plysS bacteria and purified essentially as described by Hoffmann and Roeder (1988), except that the protein was eluted from the $\mathrm{Ni}^{2+}-\mathrm{NTA}$ column (Qiagen) using $200 \mathrm{~mm}$ imidazole in BC500 [20\% glycerol, $20 \mathrm{~mm}$ Tris- $\mathrm{HCl}$ (pH 7.9), $500 \mathrm{~mm} \mathrm{KCl}, 5 \mathrm{~mm}$ DTT, $0.5 \mathrm{~mm}$ PMSF]. The eluted protein was dialyzed at $4^{\circ} \mathrm{C}$ with several changes of $\mathrm{BC} 100$ [20\% glycerol, $20 \mathrm{~mm}$ Tris- $\mathrm{HCl}(\mathrm{pH} 7.9), 100$ $\mathrm{mm} \mathrm{KCl}, 5 \mathrm{~mm}$ DTT, $0.5 \mathrm{~mm}$ PMSF] containing $50 \mu \mathrm{M} \mathrm{ZnSO}_{4}$. Typical preparations were $>75 \%$ pure as determined by Coomassie blue staining. The expression and purification of recombinant human TFIIB and human TBP (TFIID $\tau$ ) have been described in detail elsewhere (Malik et al. 1991; Takada et al. 1992). TBP was purified further using S-Sepharose resin (Pharmacia) in batch and determined to be $>99 \%$ pure by Coomassie blue staining. Mouse RXR $\beta$ (H2RIIBP or mRXR $\beta$ ) (Hamada et al. 1989) was expressed in Sf9 cells using the pBlueBac His baculovirus expression system (Invitrogen Corp.). Sf9 cells in spinner culture $\left(2 \times 10^{6}\right.$ cells $\left./ \mathrm{ml}\right)$ were infected with $6 \mathrm{His}-\mathrm{mRXR} \beta$ baculovirus at a m.o.i. of 10-20 and cultured for $60 \mathrm{hr}$. Nuclei were prepared from the infected Sf9 cells (Dignam et al. 1983), extracted with BC500, and centrifuged to pellet nuclear debris. The lysate was loaded directly on a $\mathrm{Ni}^{2+}-\mathrm{NTA}$ column and purified as described above.

\section{In vitro transcription assays}

G-less cassette assay Reactions were performed essentially as described in Pognonec and Roeder (1991), except for the following changes: Final reaction volume was $25 \mu \mathrm{l}$, contained $50 \mu \mathrm{g}$ of HeLa nuclear extract (Dignam et al. 1983), and test templates were preincubated together with TR (when indicated) for 20 min before the addition of the other components. Addition of TRIAC or the palindromic TRE oligonucleotide was as indicated in the figure legends. For single-round initiation experiments, PICs were allowed to form on templates in a reaction mixture equivalent to that described above except that NTPs were absent. Sarkosyl and NTPs were added 25 min later as indicated in Figure 6A.
Primer extension assay Transcription reactions were performed in a reaction mixture identical to the one described for G-less cassette assays except that no RNase $T_{1}$ was added and $\left[\alpha{ }^{32} \mathrm{P}\right]$ CTP was replaced with $500 \mu \mathrm{M}$ unlabeled CTP. The transcribed RNAs were analyzed by primer extension as described previously (Lillie et al. 1986). The synthetic oligonucleotide primer (5'-CAACGGTGGTATATCCAGTG-3') complementary to the coding region of the CAT gene was $5^{\prime}$ end-labeled with $\left[\gamma_{-}{ }^{32} \mathrm{P}\right] \mathrm{ATP}$ by $\mathrm{T}_{4}$ polynucleotide kinase (Boehringer Mannheim).

Reconstituted system Purification and chromatographic steps used to fractionate HeLa nuclear extract have been described in detail elsewhere (Meisterernst et al. 1991). Reconstituted transcription reactions were as described (Meisterernst et al. 1991) except for the following changes: TFIIB and TFIID fractions were replaced by recombinant TFIIB (10 ng/reaction) and recombinant TBP (10 ng/reaction). Transcripts were processed and analyzed as described above for G-less cassette assay.

\section{PIC-template purification}

Templates were preincubated with $50 \mu \mathrm{g}$ of HeLa nuclear extract in a total volume of $20 \mu \mathrm{l}$ containing transcription buffer [25 mM HEPES-KOH (pH 8.4), $7.5 \mathrm{~mm} \mathrm{MgCl}_{2}, 4 \mathrm{~mm}$ DTT, 65 $\mathrm{mM} \mathrm{KCl}, 10.5 \%$ (vol/vol) glcerol]. PIC-templates were purified by gel filtration over an $0.8-\mathrm{ml}$ packed bed of agarose beads (Bio-Gel A-1.5m, 200-400 mesh; Bio-Rad Laboratories) that were prehydrated with transcription buffer; 20 units of RNase block I (Stratagene) was then added to the eluate. Transcription of the PICs was initiated by adding a NTP mixture $(50 \mu \mathrm{M}$ each of ATP, GTP, and CTP) and $10 \mu \mathrm{Ci}$ [0.37 megaBecquerel (MBq)] of $\left[\alpha^{-32}\right.$ P]UTP. On TRE-linked HIV promoters, omission of ATP in the NTP mixture resulted in the production of a truncated 13-nucleotide transcript.

\section{Immunoprecipitations}

TR was expressed in $E$. coli in the presence of $0.1 \mathrm{mCi}$ of $\left[{ }^{35}\right.$ S]methionine per milliliter [Amersham SJ1515; $1 \mathrm{Ci}=37$ gigaBecquerel $[\mathrm{GBq})]$ and $100 \mu \mathrm{g}$ of rifampicin per milliliter. The labeled TR was purified using $\mathrm{Ni}^{2+}-\mathrm{NTA}$ column chromatography as described above. Labeled TR (50 ng) was incubated with bacterially expressed TFIIB (200 ng) or TBP (200 ng) in 25 $\mu$ l of buffer A [ $50 \mathrm{~mm} \mathrm{KCl}, 25 \mathrm{~mm}$ HEPES (pH 7.9), $6 \%$ glycerol, $2 \mu \mathrm{MnSO}_{4}, 0.2 \mathrm{mg} / \mathrm{ml}$ of BSA, $0.002 \% \mathrm{NP}-40$ ] at room temperature for $60 \mathrm{~min}$. Subsequently, the reaction volume was increased to $100 \mu \mathrm{l}$ with buffer A, and either rabbit polyclonal antisera against TFIIB (S. Malik and R.G. Roeder, in prep.) or mouse polyclonal ascites fluid against TBP (raised against recombinant TBP using standard techniques; Harlow and Lane 1988) was added. After incubation at $4^{\circ} \mathrm{C}$ with mild agitation, reaction volume was increased to $400 \mu \mathrm{l}$ with buffer $\mathrm{A}$, and $5 \mu \mathrm{l}$ (packed volume) of protein-A Sepharose (pre-equilibrated in buffer A) was added. After further incubation of the reaction mixture at $4^{\circ} \mathrm{C}$ with mild agitation, the protein A-Sepharose was pelleted by centrifugation and washed 12 times with $500 \mu l$ of buffer $\mathrm{B}$ (same as buffer $\mathrm{A}$ except $\mathrm{KCl}$ concentration was increased to $200 \mathrm{~mm}$ ). The protein A-Sepharose beads were then boiled in $2 \times$ Laemmli loading buffer and analyzed by $10 \%$ SDSPAGE. Radiolabeled protein was visualized by autoradiography after enhancement with Amplify (Amersham).

\section{Acknowledgments}

We thank Philippe Pognonec and Hiroyuki Kato for stimulating 
discussions and support; Leslie DeGroot for the gift of the human TR $\alpha$ l cDNA; Keiko Ozato for the gift of the mouse RXR $\beta$ (H2-RIIBP) cDNA; David Moore for the rGH-236 and rGH-137 templates; Koji Hisatake for preparation of TFIIB, which was used in some of the experiments; Ernest Martinez for a critical reading of this manuscript; and Camilo Parada for technical advice. J.D.F is supported by a National Research Service Award CAO9182-02 from the National Cancer Institute. A.L.R. is a Damon Runyon-Walter Winchell Cancer Research Fund postdoctoral fellow. This study was supported by National Institutes of Health grants CA42567 and AI27397 to R.G.R. and by general support from the PEW Trusts to The Rockefeller University.

The publication costs of this article were defrayed in part by payment of page charges. This article must therefore be hereby marked "advertisement" in accordance with 18 USC section 1734 solely to indicate this fact.

\section{References}

Baniahmad, A., C. Steiner, A.C. Köhne, and R. Renkawitz. 1990. Modular structure of a chicken lysozyme silencer: Involvement of an unusual thyroid hormone receptor binding site. Cell 61: 505-514.

Baniahmad, A., A.C. Köhne, and R. Renkawitz. 1992. A transferable silencing domain is present in the thyroid hormone receptor, in the v-erbA oncogene product and in the retinoic acid receptor. $E M B O$ I. 11: 1015-1023.

Beato, M. 1989. Gene regulation by steroid hormones. Cell 56: 335-344.

Berkenstam, A., M.d.M.V. Ruiz, D. Barettino, M. Horikoshi, and H.G. Stunnenberg. 1992. Cooperativity in transactivation between retinoic acid receptor and TFID requires an activity analogous to E1A. Cell 69: 401-412.

Brent, G.A., M.K. Dunn, J.W.I. Harney, T. Gulick, P.R. Larsen, and D.D. Moore. 1989a. Thyroid hormone aporeceptor represses T3-inducible promoters and blocks activity of the retinoic acid receptor. The New Biologist 1: 329-336.

Brent, G.A., J.W. Harney, Y. Chen, R.L. Warne, D.D. Moore, and P.R. Larsen. 1989b. Mutations of the rat growth hormone promoter which increase and decrease response to thyroid hormone define a consensus thyroid hormone response element. Mol. Endocrinol. 3: 1996-2004.

Brent, G.A., P.R. Larsen, J.W. Harney, R.J. Koenig, and D.D. Moore. 1989c. Functional characterization of the rat growth hormone promoter elements required for induction by thyroid hormone with and without a cotransfected $\beta$ type thyroid hormone receptor. J. Biol. Chem. 264: 178-182.

Damm, K., C.C. Thompson, and R.M. Evans. 1989. Protein encoded by v-erbA functions as a thyroid-hormone receptor agonist. Nature 339: 593-597.

Dignam, J.D., R.M Lebovitz, and R.G. Roeder. 1983. Accurate transcription initiation by RNA polymerase II in a soluble extract from isolated mammalian nuclei. Nucleic Acids Res. 11: 1475-1489.

Dynlacht, B.D., T. Hoey, and R. Tjian, 1991. Isolation of coactivators associated with the TATA-binding protein that mediate transcriptional activation. Cell 66: 563-576.

Evans, R.M. 1988. The steroid and thyroid hormone receptor superfamily. Science 240: 889-895.

Forman, B.M., C.-R. Yang, M. Au, J. Casanova, J. Ghysdael, and H.H. Samuels. 1989. A domain containing leucine zipper like motifs mediate novel in vivo interactions between the thyroid hormone and retinoic acid receptors. Mol. Endocrinol. 3: 1610-1626.
Glass, C.K., J.M. Holloway, O.V. Devary, and M.G. Rosenfeld. 1988. The thyroid hormone receptor binds with opposite transcriptional effects to a common sequence motif in thyroid hormone and estrogen response elements. Cell 54: 313323.

Glass, C.K., S.M. Lipkin, O.V. Devary, and M.G. Rosenfeld. 1989. Positive and negative regulation of gene transcription by a retinoic acid-thyroid hormone receptor heterodimer.Cell 59: 697-708.

Graupner, G., K.N. Wills, M. Tzukerman, X.-K. Zhang, and M. Pfahl. 1989. Dual regulatory role for thyroid-hormone receptors allows control of retinoic-acid receptor activity. Nature 340: 653-656.

Hamada, K., S.L. Gleason, B.-Z. Levi, S. Hirschfeld, E. Appella, and K. Ozato. 1989. H-2RIIBP, a member of the nuclear hormone receptor superfamily that binds to both the regulatory element of major histocompatibility complex class I genes and the estrogen response element. Proc. Natl. Acad. Sci. 86: 8269-8293.

Harlow, E. and D. Lane. 1988. Antibodies: A laboratory manual. Cold Spring Harbor Laboratory, Cold Spring Harbor, New York.

Hawley, D.K. and R.G. Roeder. 1985. Separation and partial characterization of three functional steps in transcription initiation by human RNA polymerase II. I. Biol. Chem. 260: 8163-8172.

- 1987. Functional steps in transcription initiation and reinitiation from the major late promoter in HeLa nuclear extract. J. Biol. Chem. 262: 3452-3461.

Hoffmann, A. and R.G. Roeder. 1991. Purification of his-tagged proteins in non-denaturing conditions suggest a convenient method for protein interaction studies. Nucleic Acids Res. 19: 6337-6338.

Hoffmann, A., E. Sinn, T. Yamamoto, J. Wang, A. Roy, M. Horikoshi, and R.G. Roeder. 1990. Highly conserved core domain and unique $\mathrm{N}$ terminus with presumptive regulatory motifs in a human TATA factor (TFIID). Nature 346: 387-390.

Hudson, L.G., J.B. Santon, C.K. Glass, and G.N. Gill. 1990. Ligand-activated thyroid hormone and retinoic acid receptors inhibit growth factor receptor promoter expression. Cell 62: $1165-1175$.

Ing, N.H., J.M. Beekman, S.Y. Tsai, M.-J. Tsai, and B.W. O'Malley. 1992. Members of the steroid hormone receptor superfamily interact with TFIIB (S300-II). I. Biol. Chem. 267: 17617-17623.

Inostroza, J.A., F.H. Mermelstein, J. Ha, W.S. Lane, and D. Reinberg. 1992. DR1, a TATA-binding protein-associated phosphoprotein and inhibitor of class II gene transcription. Cell 70: 477-489.

Jaynes, J.B. and P.H. O'Farrell. 1991. Active repression of transcription by the engrailed homeodomain protein. $E M B O J$. 10: 1427-1433.

Johnson, F.B. and M.A. Krasnow. 1992. Differential regulation of transcription preinitiation complex assembly by activator and repressor homeo domain proteins. Genes \& Dev. 6: 2177-2189.

Kato, H., H. Sumimoto, P. Pognonec, C.-H. Chen, C.A. Rosen, and R.G. Roeder. 1992. HIV-1 Tat acts as a processivity factor in vitro in conjunction with cellular elongation factors. Genes \& Dev. 6: 655-666.

Kim, H.-S., D.E. Crone, C.N. Sprung, J.B. Tillman, W.R. Force, M.D. Crew, P.L. Mote, and S.R. Spindler. 1992. Positive and negative thyroid hormone response elements are composed of strong and weak half-sites 10 nucleotides in length. Mol. Endocrinol. 6: 1489-1501.

Kliewer, S.A., K. Umesono, D.J. Mangelsdorf, and R.M. Evans. 
1992. Retinoid X receptor interacts with nuclear receptors in retinoic acid, thyroid hormone and vitamin D3 signaling. Nature 355: 446-449.

Krishna, V., K. Chatteriee, J.-K. Lee, A. Rentoumis, and J.L. Jameson. 1989. Negative regulation of the thyroid-stimulating hormone $\alpha$ gene by thyroid hormone: Receptor interaction adjacent to the TATA box. Proc. Natl. Acad. Sci. 86: 9114-9118.

Larsen, P.R., J.W. Harney, and D.D. Moore. 1986. Repression mediates cell-specific expression of the rat growth hormone gene. Proc. Natl. Acad. Sci. 83: 8283-8287.

Leid, M., P. Kastner, R. Lyons, H. Nakshatri, M. Saunders, T. Zacharewski, J.-Y. Chen, A. Staub, J.-M. Garnier, S. Mader, and P. Chambon. 1992. Purification, cloning, and RXR identity of the HeLa cell factor with which RAR or TR heterodimerizes to bind target sequences efficiently. Cell 68: 377-395.

Levine, M. and J.L. Manley. 1989. Transcriptional repression of eukaryotic promoters. Cell 59: 405-408.

Lillie, J.W., M. Green, and M.R. Green. 1986. An adenovirus Ela protein region required for transformation and transcriptional repression. Cell 40: 1043-1051.

Luckow, B. and G. Schütz. 1987. CAT constructions with multiple unique restriction sites for the functional analysis of eukaryotic promoters and regulatory elements. Nucleic Acids Res. 15: 5490.

Malik, S., K. Hisatake, H. Sumimoto, M. Horikoshi, and R.G. Roeder. 1991. Sequence of general transcription factor TFIIB and relationships to other initiation factors. Proc. Natl. Acad. Sci. 88: 9553-9557.

Marks, M.S., P.L. Hallenbeck, T. Nagata, J.H. Segars, E. Apella, V.M. Nikodem, and K. Ozato. 1992. H-2RIIBP (RXRß) heterodimerization provides a mechanism for combinatorial diversity in the regulation of retinoic acid and thyroid hormone responsive genes. EMBO J. 11: 1419-1435.

Meisterernst, M. and R.G. Roeder. 1991. Family of proteins that interact with TFIID and regulate promoter activity. Cell 67: 557-567.

Meisterernst, M., A.L. Roy, H.M. Leu, and R.G. Roeder. 1991. Activation of class II gene transcription by regulatory factors is potentiated by a novel activity. Cell 66: 981-993.

Mitchell, P.J. and R. Tjian. 1989. Transcriptional regulation in mammalian cells by sequence-specific DNA binding proteins. Science 245: 371-378.

Nakai, A., A. Sakurai, G.I. Bell, and L.J. DeGroot. 1988. Characterization of a third human thyroid hormone receptor coexpressed with other thyroid hormone receptors in several tissues. Mol. Endocrinol. 2: 1087-1092.

Pognonec, P. and R.G. Roeder. 1991. Recombinant 43-kDa USF binds to DNA and activates transcription in a manner indistinguishable from that of natural $43 / 44-\mathrm{kDa}$ USF. Mol. Cell. Biol. 11: 5125-5136.

Ptashne, M. and A.A. Gann. 1990. Activators and targets. Nature 346: 329-331.

Pugh, B.F. and R. Tjian. 1990. Mechanism of transcriptional activation by Spl: Evidence for coactivators. Cell 61: 11871197.

Ribeiro, R.C.J., P.J. Kushner, J.W. Apriletti, B.L. West, and J.D. Baxter. 1992. Thyroid hormone alters in vitro DNA binding of monomers and dimers of thyroid hormone receptors. Mol. Endocrinol. 6: 1142-1152.

Renkawitz, R. 1990. Transcriptional repression in eukaryotes. Trends Genet. 6: 192-197.

Roeder, R.G. 1991. The complexities of eukaryotic transcription initiation: Regulation of preinitiation complex assembly. Trends Biochem. Sci. 16: 402-408.
Rosen, C.A., J.G. Sodroski, and W.A. Haseltine. 1985. The location of cis-acting regulatory sequences in the human T-cell lymphotropic virus type III (HTLV-III/LAV) long terminal repeat. Cell 41: 813-823.

Samuels, H.H., B.H. Forman, Z.D. Horowitz, and Z.-S. Ye. 1988. Regulation of gene expression by thyroid hormone. 1. Clin. Invest. 81: 957-967.

Sap, J., A. Muñoz, J. Schmitt, H. Stunnenberg, and B. Vennström. 1989. Repression of transcription mediated at a thyroid hormone response element by the v-erb-A oncogene product. Nature 340: 242-244.

Sauer, F. and H. Jäckle. 1991. Concentration-dependent transcriptional activation or repression by Krüppel from a single binding site. Nature 353: 563-566.

Sawadogo, M. and R.G. Roeder. 1985. Interaction of a genespecific transcription factor with the adenovirus major late promoter upstream of the TATA box region. Cell 43: 165175.

Takada, R., Y. Nakatani, A. Hoffmann, T. Kokubo, S. Hasegawa, R.G. Roeder, and M. Horikoshi. 1992. Identification of human TFIID components and direct interaction between a $250-\mathrm{kDa}$ polypeptide and the TATA box-binding protein (TFIDT). Proc. Natl. Acad. Sci. 89: 11809-11813.

Tanese, N., B.F. Pugh, and R. Tjian. 1991. Coactivators for a proline activator purified from the multisubunit human TFIID complex. Genes \& Dev. 5: 2212-2224.

Voz, M.L., B. Peers, M.J. Wiedig, P. Jacquemin, A. Belayew, and J.A. Martial. 1992. Transcriptional regulation by triiodothyronine requires synergistic action of the thyroid receptor with another trans-acting factor. Mol. Cell. Biol. 12: 39913997.

Yu, V.C., C. Delsert, B. Anderson, J.M. Holloway, O.V. Devary, A.M. Näär, S.Y. Kim, J.-M. Boutin, C.K. Glass, and M.G. Rosenfeld. 1991. RXRß: A coregulator that enhances binding of retinoic acid, thyroid hormone, and vitamin $\mathrm{D}$ receptors to their cognate response elements. Cell 67: 1251-1266.

Zawel, L. and D. Reinberg. 1992. Advances in RNA polymerase II transcription. Curr. Opin. Cell Biol. 4: 488-495.

Zhang, X.-k., B. Hoffman, P.B.-V. Tran, G. Graupner, and M. Pfahl. 1992. Retinoid X receptor is an auxiliary protein for thyroid hormone and retinoic acid receptors. Nature 355: 441-445.

Zhou, Q., P.M. Lieberman, T.G. Boyer, and A.J. Berk. 1992. Holo-TFIID supports transcriptional stimulation by diverse activators and from a TATA-less promoter. Genes \& Dev. 6: 1964-1974. 


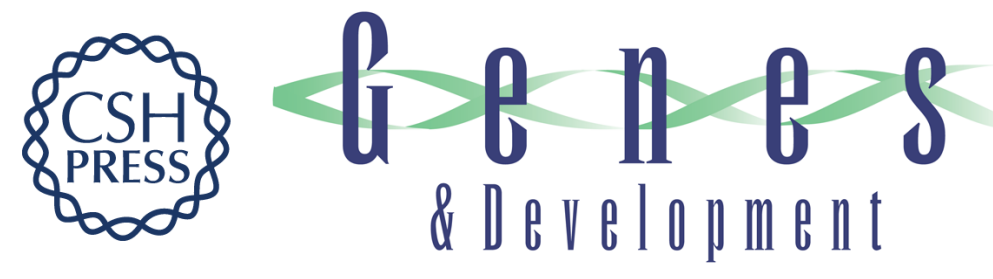

\section{Unliganded thyroid hormone receptor inhibits formation of a functional preinitiation complex: implications for active repression.}

J D Fondell, A L Roy and R G Roeder

Genes Dev. 1993, 7:

Access the most recent version at doi:10.1101/gad.7.7b.1400

References This article cites 58 articles, 16 of which can be accessed free at:

http://genesdev.cshlp.org/content/7/7b/1400.full.html\#ref-list-1

License

Email Alerting

Service

Receive free email alerts when new articles cite this article - sign up in the box at the top right corner of the article or click here.

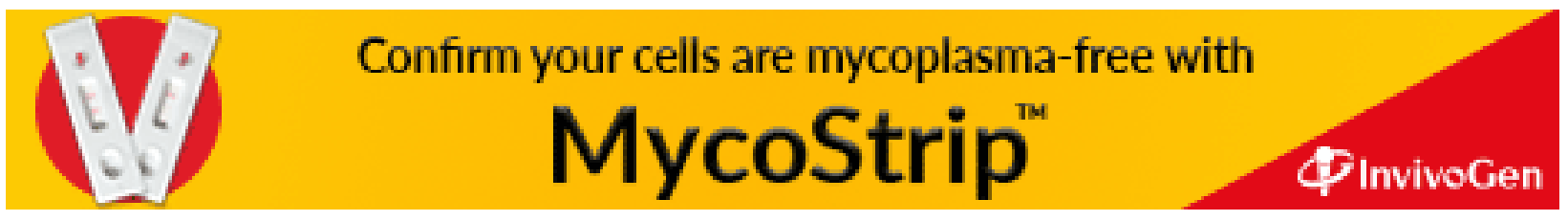

